\title{
Tempo-spatial distribution of air pollution index in Nanning city*
}

\author{
Ping Chen, Xin-Ying Zhang ${ }^{\dagger}$ Jiao Chen, Ni-Yu Wei and Shan-Chun Lin \\ Key Laboratory of Environment Change and Resources \\ Use in Beibu Gulf Ministry of Education, \\ Guangxi Teachers Education University, \\ Nanning, Guangxi 530001, China \\ tE-mail:191957125@qq.com \\ www.gxtc.edu.cn
}

\begin{abstract}
Spatial statistical analysis and wavelet analysis are used to study the air pollution index (API) of Nanning city according to the daily data from Jan 1, 2009 to Dec 31, 2012 and monthly air quality data from 2009 to 2013 . The results show that the Tempo-spatial distribution characteristics of API in Nanning appear like this: the API in the South and North is more serious than East and West; and air pollution is more serious in autumn and winter than spring and summer. The API varies periodically. The cycle period of daily variations of API is about 290 days.
\end{abstract}

Keywords: Air Pollution Index (API); Spatial Statistical Analysis; Wavelet Analysis.

\section{Introduction}

In recent years, air pollution has become one of the most concerning environmental problems in the world. The management of air quality has also become one of the most difficult matters in government departments. The domestic and foreign scholars have carried out a lot of researches on the changes of urban air quality, but these studies are mainly focused on the compositions of air pollutants, the impact factors and so on [1-3]. A World Bank study reported that 16 out of the top 20 most-polluted cities in the world were located in China [4]. Only one percent of the 560 million Chinese urban dwellers breathed clean air considered safe by European Union standards [5].

Nanning, the capital city of Guangxi Zhuang Autonomous Region, is the political, economic and transportation center of Guangxi. The air quality of Nanning has received much attention. This paper, based on the analysis of the

\footnotetext{
* This work is supported by National Natural Science Foundation of China (41461095).

$\dagger$ Work partially supported by Guangxi Elite Hill land of Soil Pollution and Ecological Restoration and the Collaborative Innovation Center of the Ecological Environment \& Integration Development in the Xijiang River Basin.
} 
spatial and temporal distribution characteristics of air pollution in Nanning city, makes full use of the ArcGIS and MATLAB software to carry out spatial statistical analysis and wavelet analysis and explores the characteristics of the spatial distribution, inter-annual variation and seasonal variation of the air pollution index (API).

\section{Data and Methods}

\subsection{Air pollution index data}

The daily API values from January 1, 2009 to December 31, 2012 in Nanning are downloaded from the website of the Ministry of Environmental Protection of the People's Republic of China. And the monthly air quality data of eight monitoring sites for a 5-year period from 2009 to 2013 are obtained from the website of Environmental Protection Bureau of Nanning. These data include API and primary pollutant. The eight special monitoring sites are Xianhu, Zhenninghuayuan, Beihu, City Monitoring Station, Autonomous agricultural school, Yinghuajiayuan, Daziranhuayuan, and Shajing Street. The monitoring site distribution diagram is shown in Figure 1.

\subsection{Methods}

This paper uses the spatial interpolation of ArcGIS and the wavelet analysis program of MATLAB to analyze the air pollution index. Spatial analysis is based on the analysis of the form of spatial data and the location of the geographic object, its function is to extract and transmit spatial information. Spatial analysis ability, especially the extraction and transmission capability of spatial information, is the main aspect of GIS, which is different from the general information system.

Wavelet transform is a local transformation, but it can decompose the signal at any time frequently in order to extract useful information effectively [6].Wavelet analysis is applied to many practical applications, and it is suitable for dealing with the vast majority of the non stability signal, which is different from the common Fourier transform [7].The wavelet transform has a good adaptive character and frequent distinctive function, which can focus on the arbitrary details of the signal, so as to observe the changes in the different time scales.

Morlet wavelet is widely used in the analysis of time series. It can be used to identify the periodic and random fluctuations [8]. 


\section{Spatial Characteristics of Air Pollution Index in Nanning City}

\subsection{Spatial analysis of air pollution index in Nanning City}

The Figure 2, Distribution of annual average value of API in Nanning city, is obtained by interpolation of the API of each monitoring site. In general, the average annual air quality level in Nanning is grade II, the quality of the situation reached a good level. From Figure 2, it is obvious that the API in the South and North is more serious than in the East and West of Nanning, which takes the Shajing Street as the representative of the lowest API, while the highest value is in the Youai-Beihu area. The old city zone is located in the Midwest of the north shore of the Yongjiang River, which the high API value was high. According to the "Nanning urban ambient air quality function zoning", Langdong and Liangfengjiang River Forest Park were distributed in the East and southwest of urban areas, which was the designated to be the first functional region [9]. Therefore, the API value in here is much lower.

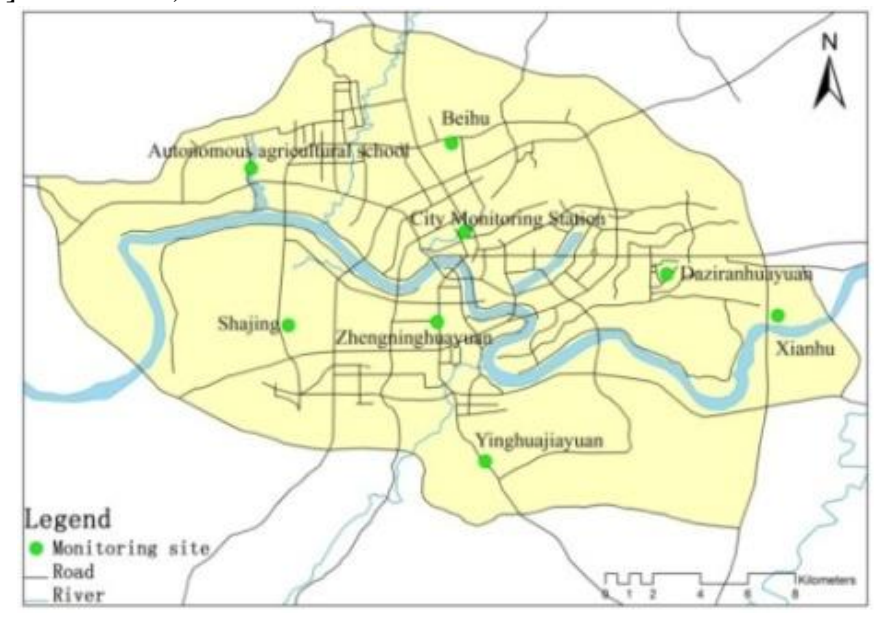

Fig. 1. Air monitoring site of Nanning city. 


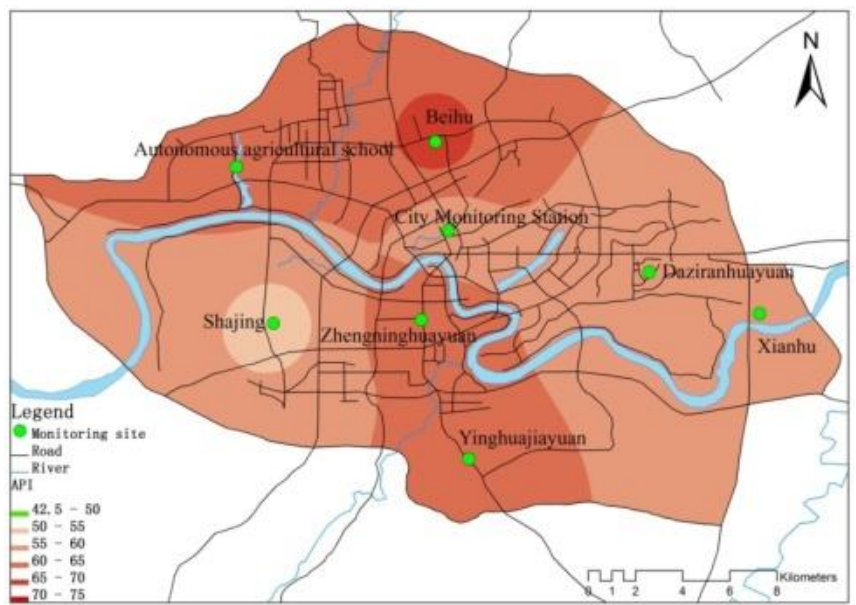

Fig. 2. Distribution of annual average value of API in Nanning city.

\subsection{Analysis of the characteristics of the four seasons of API}

During the spring, the majority of API was below 60 mostly, and from the overall point of view API reached a good level. The high value zone of API was located in the northern part of Nanning City, which was Xixiangtang-Daxue road area and Youai-Beihu area. The API of Shajing area which was located in the southwest of Nanning and the ASEAN business area and Xianhu area which were located in the east of the city had the lower API value. The air quality at the perimeter of the Shajing monitoring point could be achieved by the optimal level.API was the lowest in the four seasons in the summer. It reached the optimal level generally. Only in the Youai-Beihu area, API had a relative high value has a relatively high value area.API was higher in autumn and winter than in spring and summer. Compared with the winter, the high value range of autumn was relatively large. Similarly, the highest value area was located in the Youai-Beihu area; the lower value of the district was located in the East and west of the city. Although the maximum value of API reached 75, it still belonged to a good level. Therefore, the air quality in Nanning was good.

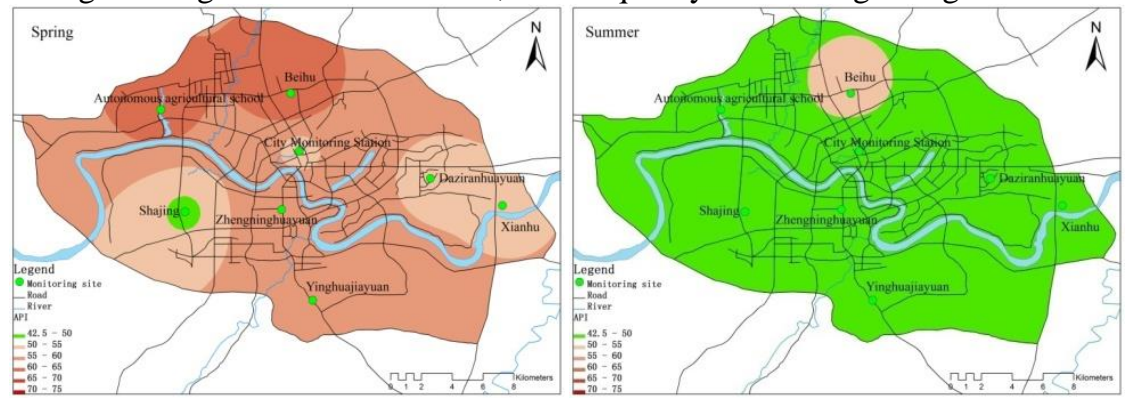




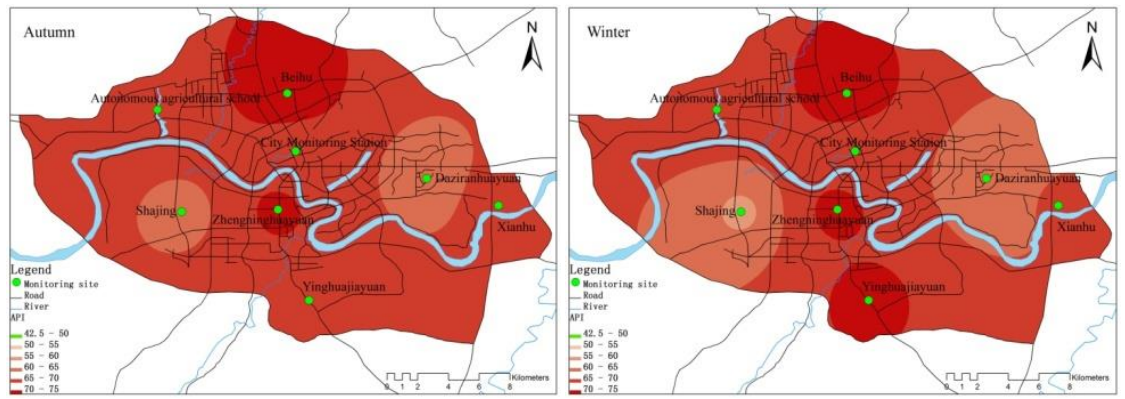

Fig. 3. Distribution of seasonal average value of API.

\section{Time Variation Characteristics of Air Pollution Index}

API data is a time series of daily data, which is influenced by many factors. Direct analysis of the original data is difficult to find the regularity of the annual change. If the average classification method is used, though it is simple, some details would be missing. Therefore, it is necessary to find a reasonable analysis method. Wavelet analysis is a time-frequency analysis method, which can effectively analyze the time variation characteristics of API. And it has been studied that it is feasible to study the characteristics of API by using wavelet analysis [11].

The wavelet analysis program of MATLAB is used to analyze the API of Nanning city according the daily data for a 4-year period from Jan 1, 2009 to Dec 31, 2012, the following figure was appeared.

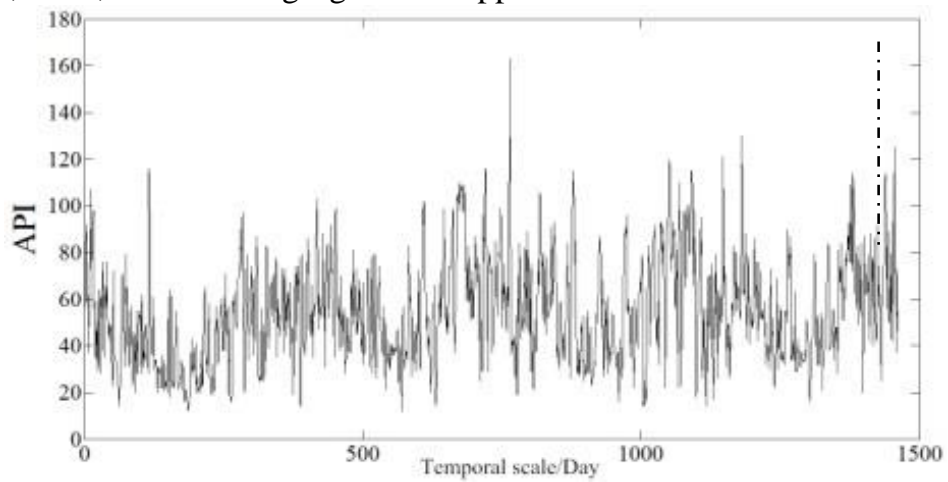

Fig. 5. Time sequence diagram of the API in Nanning. 


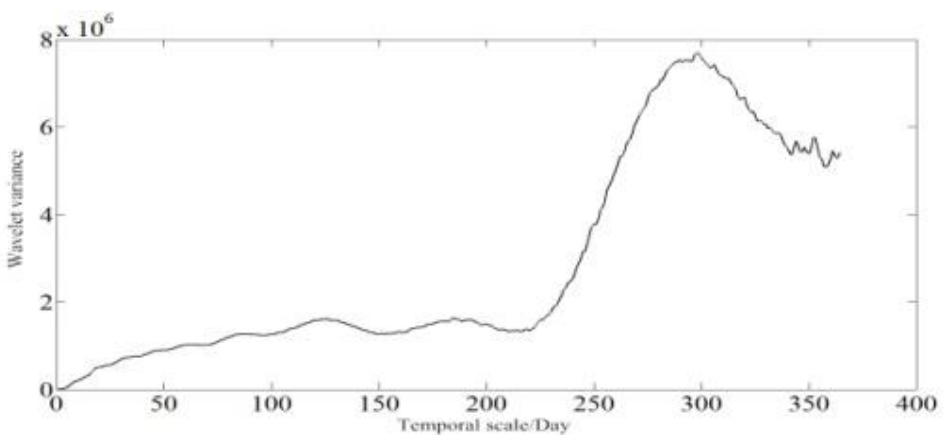

Fig. 6. Wavelet variance analysis

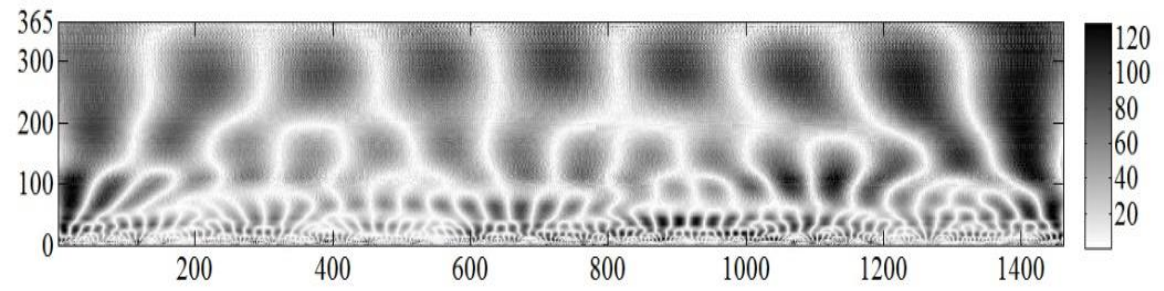

Fig. 7. Morlet wavelet transform.

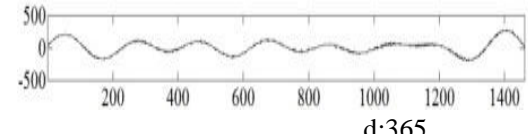

$\mathrm{d}: 365$

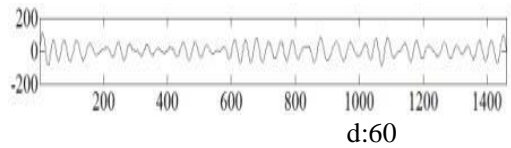

Fig. 8. Wavelet coefficients of API at some scales.

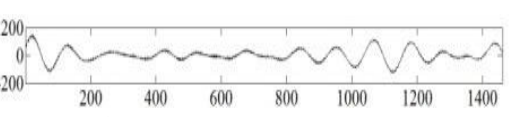

$\mathrm{d}: 180$

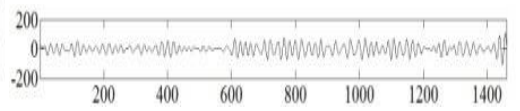
$\mathrm{d}: 30$

Figure 5 is the time sequence diagram of the API in Nanning according the daily data for a 4-year period from Jan 1, 2009 to Dec 31, 2012. Figure 6 and Figure 8 are the result of the wavelet analysis of the API in Nanning. Through the wavelet variance diagram, it could be seen that the main period of the change of air pollution index in Nanning was about 290d. Figure 7 shows the Morlet wavelet transform which time scale was 365 d. Figure 7 clearly showed the characteristics of periodic oscillations and abrupt point of the API in the different time scales of Nanning city. In the picture, the signal oscillation is expressed by the gray level; the greater the intensity of the gray scale indicates that the API was higher than the average of the year. More abundant information of the periodic scale could be obtained from Figure 7. By Figure 7, there is a phenomenon of nested complex structures with multiple time periods. The short 
period of $300 \mathrm{~d}$ and $150 \mathrm{~d}$ is clearly visible. This conclusion could be confirmed from Figure 8.

\section{Conclusions}

Through the analysis of the daily API data from 2009 to 2012 and monthly air quality data from 2009 to 2013, the following conclusions are obtained in this paper.

1. In general, the distribution of API in the main city of Nanning has the following characteristics: high value zone is located in the city of the north, with the second highest in the southern, while the low value area is mainly located in the East and west sides of the city, the API in the South and North is more serious than in the East and West. The air quality can reach good level.

2. From the seasonal point of view, the distribution of high value regions in different seasons is basically the same, and they are in the Youai-Beihu area. From the pollution index point of view, the air quality in summer is best, and then is the spring, autumn and winter is poor.

3. The change of air pollution index in Nanning city has a significant periodicity by using wavelet analysis to discuss and analyze the API of Nanning, and the main period is about $290 \mathrm{~d}$.

\section{References}

1. L. Zhang, C.Y. Fu, X.J. Zheng, Study on air pollution feature and its effect in Guangzhou city. Ecology and Environment, 2nd, pp.305-308(2007).

2. J.J. Zhang, S.Q. Wei, Current Status and Characteristics of Urban Air Pollution in China. Sichuan Environment, 3rd, pp.104-108+112(2006).

3. X.F. Li, M.J. Zhang, S.J. Wang, Variation Characteristics and Influencing Factors of Air Pollution Index in China. Environmental Science, 6th, pp.1936-1943(2012).

4. R.E. López, V. Thomas, The Quality of Growth: Fiscal Policies for Better Results. The World Bank, Washington, D.C, 2008.

5. J. Kahn, J. Yardley, As China Roars, Pollution Reaches Deadly Extremes. The New York Times. August 26, 2007.

6. Z. Gao, H.X. Yu, Matlab wavelet analysis and Application. Beijing: National Defense Industry Press, pp.1-28(2007).

7. H. Janicke, M. Bottinger, Visual exploration of climate variability changes using wavelet analysis. IEEE Trans, 15th, 1375-382(2009).

8. Y. Huang, Urban air quality functional area divided into two categories .Nanning Daily 2007-11-29005. 
9. H.B. Xie, Analysis on Weather Factors Affecting on Atmosphere Quality in Nanning City. Yunnan Environmental Science, S1, pp.28-31(2005).

10. M. Cao, X.C. Lin, G. Liu, Application of Mallat Wavelet Deposition in Air Pollution Index Analyses. China Environmental Protection Industry, 10(1), pp.13-17(2006). 